\title{
UNCOVERING ULTRA-LUMINOUS GALAXIES \\ IN THE IRAS FSC THROUGH RADIO AND OPTICAL \\ CROSS-IDENTIFICATION
}

\author{
G. ALDERING \\ University of Minnesota, Department of Astronomy
}

\section{Introduction}

Ultraluminous infrared galaxies (ULIRGs) have luminosities $\left(10^{12} L_{\odot}\right)$ once exclusive to QSOs. This suggests they might be the early, dust-enshrouded stages of QSOs. ULIRGs have $\sim 3.5 \times$ the space density of QSOs at the present epoch. Quasars reached their peak space density at $z \sim 2$, so if ULIRGs are QSO precursors, there should be a dramatic increase in their space density up to $z \sim 2$. The small number of known ULIRGs makes it difficult to explore links between ULIRGs and QSOs, much less measure their evolution. To do so, a large sample of ULIRG candidates must be identified. The IRAS FSC contains $\sim 60,000$ probable galaxies, of which $1 \%-3 \%$ should have $L_{F I R}>10^{12} L_{\odot}$. We discuss an efficient and reliable method which uses IRAS-VLA-APS cross-identification and flux ratios to mine the FSC for likely ULIRGs.

\section{Exploiting the $L_{F I R}$ versus $F_{F I R} / F_{\text {opt }}$ Correlation}

Likely ULIRGs can be identified by using the strong correlation between $F_{F I R} / F_{\text {opt }}$ and $L_{F I R}$, as shown in Figure 1 . Since the IRAS positions are too crude for a direct cross-identification, the radio-FIR correlation (see below) was exploited using the $N V S S$ (Condon, this volume, p. 19) to refine the positions. Fits to the forward and inverse relation are shown as dashed lines. The inverse relation has $\sigma \sim 0.22$ dex once outliers are rejected. Among the sub-sample of 64 IRAS galaxies with $F_{F I R} / F_{\text {opt }}>10^{1.2}, 18$ of 22 ULIRGs would be found. This translates to an efficiency of $\sim 30 \%$ and a completeness of $\sim 80 \%$. 


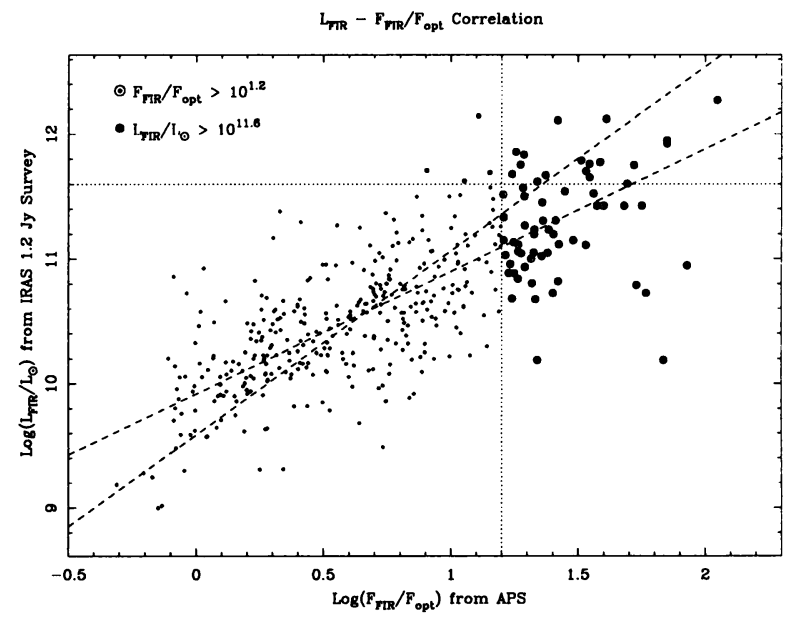

Figure 1. The $F_{F I R} / F_{\text {opt }}-L_{F I R}$ relation constructed from sources matched between the IRAS 1.2 Jy survey, the $N V S S 1.49 \mathrm{GHz}$ radio catalog, and the available $A P S$ catalogs. The forward and inverse unweighted least-squares fits are shown. The solid symbols highlight those galaxies with $L_{F I R}>10^{11.6} L_{\odot}$. The concentric circles highlight ULIRG candidates selected using $F_{F I R} / F_{\text {opt }} \geq 10^{1.2}$. These cuts have been used to estimate the efficiency and completeness of the $F_{F I R} / F_{\text {opt }}$-excess selection technique.

\section{Increasing Reliability using the Radio-FIR Correlation}

Determining reliable optical identifications for sources with large $F_{F I R} / F_{\text {opt }}$ is plagued by the large size of the IRAS error ellipse at the limit of the FSC $\left(\sim 7^{\prime \prime} \times 25^{\prime \prime}\right)$ relative to the surface density of faint optical candidates. The presence of IR cirrus further complicates the identification of faint $60 \mu \mathrm{m} I R A S$ sources. The strong correlation between radio continuum emission and FIR flux, $L_{1.49 G H z}=10^{11.379 \pm 0.004}\left(L_{F I R} / L_{\odot}\right)^{1.030 \pm 0.014}$ with $\sigma \sim 0.18$ dex determined from the Figure 1 dataset, enables confirmation and positional refinement of faint FIR sources.

\section{Application}

We are performing cross-identification of sources from the IRAS FSC as the corresponding radio (NVSS \& FIRST) and optical (POSS I \& POSS II) data become available. The entire sky with $\delta>-33^{\circ}$ and $|b|>20^{\circ}$ is being processed. ULIRGs are out there waiting to be found; in related work, we have recently discovered one ULIRG at $z=1.1$, and two at $z=1.3$ from spectroscopic follow-up of sources in the IRAS VFSS. These were found in relatively small samples, suggesting an excess of high redshift ULIRGs, as expected if ULIRGs evolve like QSOs. 